\title{
Comment on "Effects of cosmic-string framework on the thermodynamical properties of anharmonic oscillator using the ordinary statistics and the $q$-deformed superstatistics approaches"
}

\author{
Francisco A. Cruz Neto ${ }^{a}$, Luis B. Castro ${ }^{b}$ \\ Departamento de Física, Universidade Federal do Maranhão, Campus Universitário do Bacanga, São Luís, MA 65080-805, Brazil
}

Received: 13 February 2018 / Accepted: 25 April 2018 / Published online: 14 June 2018

(C) The Author(s) 2018

\begin{abstract}
We point out a misleading treatment in a recent paper published in this Journal (Sobhani et al., Eur Phys J C 78:106, 2018) regarding solutions for the Schrödinger equation with a anharmonic oscillator potential embedded in the background of a cosmic string mapped into biconfluent Heun equation. This fact jeopardizes the thermodynamical properties calculated in this system.
\end{abstract}

In a recent paper in this Journal, Sobhami et. al. [1] have studied the thermodynamical properties of the anharmonic oscillator within cosmic-string framework using ordinary statistic and the $q$-deformed superstatistics approaches. To achieve their goal, the authors need to calculate the wave function and the energy spectrum, which have been obtained from the Schrödinger equation within a cosmic-string framework mapped into biconfluent Heun differential equation. It is worthwhile to mention that all results depend mainly on the energy spectrum of the system. The purpose of this comment is point to out a misleading treatment on the solution of the biconfluent Heun equation, this fact jeopardizes the results of [1].

The time-independent Schrödinger equation with an anharmonic oscillator potential embedded in the background of a cosmic string is given by

$$
\begin{aligned}
& \frac{d^{2} \Phi(\rho)}{d \rho^{2}}+\frac{1}{\rho} \frac{d \Phi(\rho)}{d \rho} \\
& +\left(\varepsilon-k_{z}^{2}-\left(a_{v}+l^{2} \alpha^{2}\right) \rho^{2}-b_{v} \rho^{4}-c_{v} \rho^{6}\right) \Phi(\rho)=0 .
\end{aligned}
$$

Redefining the wave function as $\Phi(\rho)=\frac{R(\rho)}{\sqrt{\rho}}$, one can remove the first derivative and rewrite Eq. (1) as

\footnotetext{
a e-mail: alvesfcn@gmail.com

be-mails: luis.castro@ufma.br; luis.castro@pq.cnpq.br
}

$$
\begin{aligned}
& \frac{d^{2} R(\rho)}{d \rho^{2}}+\left(\varepsilon+\frac{1}{4 \rho^{2}}-k_{z}^{2}-\left(a_{v}+l^{2} \alpha^{2}\right) \rho^{2}\right. \\
& \left.-b_{v} \rho^{4}-c_{v} \rho^{6}\right) R(\rho)=0 .
\end{aligned}
$$

Making use of the new variable $y=\rho^{2}$ and redefining the wave function as $R(y)=\frac{f(y)}{\sqrt[4]{y}}$, the Eq. (2) becomes

$$
\frac{d^{2} f(y)}{d y^{2}}+\left(\kappa+\frac{A}{y}-B y-C y^{2}+\frac{1 / 4}{y^{2}}\right) f(y)=0,
$$

where

$$
\begin{aligned}
\kappa & =-\frac{1}{4}\left(a_{v}+l^{2} \alpha^{2}\right), \\
A & =\frac{1}{4}\left(\varepsilon-k_{z}^{2}\right), \\
B & =\frac{b_{v}}{4}, \\
C & =\frac{c_{v}}{4} .
\end{aligned}
$$

The solution for the differential Eq. (3) with $C$ necessarily real and positive $\left(c_{v}>0\right)$, is the solution of the Schrödinger equation for the three-dimensional harmonic oscillator plus a Cornell potential [2-4]. It is worthwhile to mention that Refs. [2,3] present some erroneous calculations.

Considering the solution in the form of [3]

$$
f(y)=y^{1 / 2} \exp \left(-\frac{\sqrt{c_{v}}}{4} y^{2}-\frac{b_{v}}{4 \sqrt{c_{v}}} y\right) F(y),
$$

and by introducing the new variable and parameters:

$$
\begin{aligned}
x & =\left(\frac{c_{v}}{4}\right)^{1 / 4} y, \\
\beta^{\prime} & =\left(\frac{4}{c_{v}}\right)^{3 / 4} \frac{b_{v}}{4}, \\
\gamma^{\prime} & =\left(\frac{4}{c_{v}}\right)^{3 / 2}\left[\frac{b_{v}^{2}}{64}-\frac{c_{v}}{16}\left(a_{v}+l^{2} \alpha^{2}\right)\right],
\end{aligned}
$$


one finds that the solution can be expressed as a solution of the biconfluent Heun differential equation [5-9]

$$
\begin{gathered}
\frac{d^{2} F(x)}{d x^{2}}+\left(\frac{1}{x}-\beta^{\prime}-2 x\right) \frac{d F(x)}{d x} \\
+\left[\gamma^{\prime}-2-\frac{\Theta}{x}\right] F(x)=0,
\end{gathered}
$$

with

$\Theta=\frac{1}{2}\left[\delta^{\prime}+\beta^{\prime}\right]$,

where

$\delta^{\prime}=\left(\frac{4}{c_{v}}\right)^{1 / 4} \frac{k_{z}^{2}-\varepsilon}{2}$.

This differential equation has a regular singularity at $x=0$ and an irregular singularity at $x=\infty$. The regular solution at the origin is given by

$H_{b}\left(0, \beta^{\prime}, \gamma^{\prime}, \delta^{\prime} ; x\right)=\sum_{j=0}^{\infty} \frac{1}{\Gamma(1+j)} \frac{A_{j}}{j !} x^{j}$,

here $\Gamma(z)$ is the gamma function, $A_{0}=1, A_{1}=\Theta$ and the remaining coefficients for $\beta^{\prime} \neq 0$ satisfy the recurrence relation,

$$
A_{j+2}=\left[(j+1) \beta^{\prime}+\Theta\right] A_{j+1}-(j+1)^{2}(\Delta-2 j) A_{j},
$$

where $\Delta=\gamma^{\prime}-2$.

The series is convergent and tends to $\exp \left(x^{2}+\beta^{\prime} x\right)$ as $x \rightarrow \infty$. It is true that the presence of $\exp \left(x^{2}+\beta^{\prime} x\right)$ in the asymptotic behavior of $H_{b}\left(0, \beta^{\prime}, \gamma^{\prime}, \delta^{\prime} ; x\right)$ perverts the normalizability of the solution $f(y)$, i.e $f(y) \propto$ $\exp \left(\frac{\sqrt{c_{v}}}{4} y^{2}+\frac{b_{v}}{4 \sqrt{c_{v}}} y\right)$ as $y \rightarrow \infty$. Nevertheless, this trouble can be surpassed by considering a polynomial solution for $H_{b}\left(0, \beta^{\prime}, \gamma^{\prime}, \delta^{\prime} ; x\right)$. In fact, $H_{b}\left(0, \beta^{\prime}, \gamma^{\prime}, \delta^{\prime} ; x\right)$ presents polynomial solutions of degree $n$ if and only if two conditions are satisfied:

$\Delta=2 n, \quad n=0,1,2, \ldots$

and

$A_{n+1}=0$.

Now, the condition (18) provides a polynomial of degree $n+1$ in $\delta^{\prime}$ and there are at most $n+1$ suitable values of $\delta^{\prime}$. Therefore, the energy eigenvalues of the system are obtained for the both conditions (17) and (18).

From the condition (17), one obtains

$b_{v, n}= \pm\left(\frac{c_{v}}{4}\right)^{3 / 4} \sqrt{128(n+1)+\sigma}$,

where

$\sigma=16\left(\frac{4}{c_{v}}\right)^{1 / 2}\left(a_{v}+l^{2} \alpha^{2}\right)$.
This result shows a constraint that involves a specific value for $b_{v}$ as a function of $n, c_{v}, a_{v}, l$ and $\alpha$. The problem does not end here, it is necessary to analyze the second condition of quantization.

Now, we focus attention on the condition (18). For $n=0$, the condition $A_{1}=\Theta=0$ results in an algebraic equation of degree one in $\delta^{\prime}$ :

$\delta^{\prime}+\beta^{\prime}=0$,

which furnishes the following expression for the energy

$\varepsilon_{0}=k_{z}^{2}+\frac{b_{v, 0}}{2}\left(\frac{4}{c_{v}}\right)^{1 / 2}$.

Note that this result presents two different classes of solutions that depend on the sign of $b_{v, 0}$. If $A \gtrless 0$ [Eq. (5)], that implies $\varepsilon \gtrless k_{z}^{2}$, then $b_{v, 0} \gtrless 0$, so we get

$\varepsilon_{0, \pm}=k_{z}^{2} \pm \frac{1}{2}\left(\frac{c_{v}}{4}\right)^{1 / 4} \sqrt{128+\sigma}$.

The expression (22) represents the energy eigenvalue for $n=$ 0 .

Now, let us consider the case for $n=1$, which implies that $A_{2}=\left(\beta^{\prime}+\Theta\right) \Theta-2=0$. In this case we obtain an algebraic equation of degree two in $\delta^{\prime}$ :

$\left(\delta^{\prime}+\beta^{\prime}\right)^{2}+2 \beta\left(\delta^{\prime}+\beta^{\prime}\right)-8=0$,

which furnishes the energy eigenvalues

$\varepsilon_{1,1}=k_{z}^{2}+\left(\frac{4}{c_{v}}\right)^{1 / 2}\left[b_{v, 1}+\frac{1}{2} \sqrt{\left(b_{v, 1}\right)^{2}+128\left(\frac{c_{v}}{4}\right)^{3 / 2}}\right]$

and

$\varepsilon_{1,2}=k_{z}^{2}+\left(\frac{4}{c_{v}}\right)^{1 / 2}\left[b_{v, 1}-\frac{1}{2} \sqrt{\left(b_{v, 1}\right)^{2}+128\left(\frac{c_{v}}{4}\right)^{3 / 2}}\right]$.

For simplicity, we will only consider the case $b_{v, 1}>0$ [positive sign in (19)]. Therefore, the energy eigenvalues in this case are given by

$\varepsilon_{1,1,+}=k_{z}^{2}+\left(\frac{c_{v}}{4}\right)^{1 / 4}\left[\sqrt{\sigma+256}+\frac{1}{2} \sqrt{\sigma+384}\right]$

and

$\varepsilon_{1,1,+}=k_{z}^{2}+\left(\frac{c_{v}}{4}\right)^{1 / 4}\left[\sqrt{\sigma+256}-\frac{1}{2} \sqrt{\sigma+384}\right]$.

For the case of $n=2$, the condition $A_{3}=0$ results in an algebraic equation of degree three in $\delta^{\prime}$. And the solutions, in fact, turn out to be three values for the energy (considering $b_{v, 3}>0$ ). For $n \geq 2$ the algebraic equations are cumbersome. With this result, we conclude that the energy can not be labeled with $n$. This is a peculiar behavior of the biconfluent Heun equation. Our results show that the expression for the 
energy in Ref. [1] is wrong, probably due to erroneous calculations in the manipulation of the biconfluent Heun equation. The correct quantization condition is obtained applying two conditions: the condition (17) is used to obtain constraints between the potential parameters and the condition (18) is used to obtain the expression of energy eigenvalues. It is worthwhile mention that condition of quantization is different for each value of $n$.

In summary, we analyzed the solution for the Schrödinger equation with a anharmonic oscillator potential embedded in the background of a cosmic string. In this process, the problem is mapped into biconfluent Heun differential equation and using appropriately the conditions (17) and (18), we found the correct energy eigenvalues and constraints on the potential parameters. Also, we showed that there is no need for fixing the value of $c_{v}=4$ for obtain a biconfluent Heun equation, in contrast to Ref. [1]. Additionally, the thermodynamical properties calculated in Ref. [1] depend of the energy spectrum relation, therefore our results jeopardize the main results of Ref. [1].

Acknowledgements We acknowledge valuable comments from the anonymous referee. This work was supported in part by means of funds provided by CNPq, Brazil, Grant No. 307932/2017-6 (PQ).

Open Access This article is distributed under the terms of the Creative Commons Attribution 4.0 International License (http://creativecomm ons.org/licenses/by/4.0/), which permits unrestricted use, distribution, and reproduction in any medium, provided you give appropriate credit to the original author(s) and the source, provide a link to the Creative Commons license, and indicate if changes were made.

Funded by SCOAP ${ }^{3}$.

\section{References}

1. H. Sobhani, H. Hassanabadi, W.S. Chung, Eur. Phys. J. C 78(2), 106 (2018). https://doi.org/10.1140/epjc/s10052-018-5581-y

2. B. Leaute, G. Marcilhacy, J. Phys. A: Math. and Gen. 19(17), 3527 (1986). http://stacks.iop.org/0305-4470/19/i=17/a=017

3. A. Ronveaux, Heun's differential equations (Oxford University Press, Oxford, 1986)

4. L.B. Castro, Phys. Rev. C 86, 052201 (2012). https://doi.org/10. 1103/PhysRevC.86.052201

5. E.R. Figueiredo Medeiros, E.R. Bezerra de Mello, Eur. Phys. J. C 72(6), 2051 (2012). https://doi.org/10.1140/epjc/ s10052-012-2051-9

6. K. Bakke, F. Moraes, Phys. Lett. A 376(45), 2838 (2012). https:// doi.org/10.1016/j.physleta.2012.09.006

7. K. Bakke, Ann. Phys. (N.Y.) 341, 86 (2014). https://doi.org/10.1016/ j.aop.2013.11.013

8. K. Bakke, C. Furtado, Ann. Phys. (N.Y.) 355, 48 (2015). https://doi. org/10.1016/j.aop.2015.01.028

9. R.L.L. Vitória, C. Furtado, K. Bakke, Eur. Phys. J. C 78(1), 44 (2018). https://doi.org/10.1140/epjc/s10052-018-5524-7 